\title{
Cuerpo de contenidos para la formación de Controlador de Tránsito Aéreo en el ámbito de las Ciencias Aeronáuticas en Honduras
}

Alex Matamoros

\section{Resumen}

Este artículo presenta las partes principales de un trabajo preparado para adaptar un Técnico en Controlador de Tránsito Aéreo (ATC) a la Licenciatura en Ciencias Aeronáuticas que actualmente (diciembre de 2014) se encuentra en proceso de aprobación por parte de la Autoridades Académicas de la UNAH. En una primera parte se proponen los objetivos a alcanzar con la investigación realizada, los cuales buscan básicamente definir los contenidos que debería incluir una propuesta de Técnico ATC con nivel universitario. Seguidamente se explica la metodología de consulta utilizada. Los resultados presentados en la tercera parte son cuatro: 1. El espectro de posibilidades de empleo que tiene el control de tránsito aéreo en la Agencia Hondureña de Aeronáutica Civil (AHAC) representado en los perfiles de puesto del Departamento de Navegación Aérea de esta agencia; 2. Un repaso de la composición de la carrera de Técnico Aeronáutico ATC u otras carreras similares en distintos Centros de Formación Superior, 3. Un resumen de las opiniones de distintos profesionales aeronáuticos sobre la formación y el desempeño de los ATC en Honduras, 4. La composición de las asignaturas que incluiría un Técnico ATC propuesto desde el Departamento de Ciencias Aeronáuticas de FACES. Se finaliza la exposición con una serie de recomendaciones encaminadas a fortalecer las funciones formativas de la Agencia Hondureña de Aviación Civil y de la UNAH, en su condición de instituciones del Estado en materia aeronáutica y formación superior respectivamente.

Palabras Clave: Control de Tráfico Aéreo, perfil de puesto, asignaturas, propuesta formativa

\section{Abstract}

This article presents the main parts of a paper prepared for adapting a Technician Air Traffic Controller (ATC) for a degree in Aeronautical Sciences wich is currently 
(December 2014) in the process of approval by the Academic Authorities of the UNAH . The first part presents the objectives to achieve with this research, which seek basically to define the contents should include a proposal for ATC Technician with university level. Following consultation methodology used is explained. The results presented in the third part are four: 1 . The spectrum of employment opportunities has control of air traffic in the Honduran Civil Aviation Agency (HCAA) represented in the job profiles of the Department of Air Navigation agency; 2. A review of the composition of the career of Aeronautical Technical ATC or other similar in various centers of College Education, 3. A summary of the views of other aviation professionals on the formation and performance of ATC in Honduras, 4. the composition of the subjects that include an ATC Technical proposed by the Department of Aeronautical Sciences of FACES/UNAH. The presentation ends with a series of conclusions aimed at strengthening the educational functions of the Honduran Civil Aviation Agency and the UNAH, in their functions as state institutions in aeronautics and higher education respectively.

Keywords: Air Traffic Control, job profile, assignments, formative proposal.

Alex Matamoros, (algemaca@hotmail.com ),Departamento de Ciencias Aeronáuticas Facultad de Ciencias Espaciales, Universidad Nacional Autónoma de Honduras - UNAH 


\section{Introducción}

La preocupación principal del Departamento de Ciencias Aeronáuticas al proponer esta investigación es la de dar al Controlador de Tránsito Aéreo hondureño un estatus de profesional universitario. Para el logro de tal propósito es necesaria, pero no suficiente una propuesta de currículo para ATC, a esta propuesta se debe sumar otros elementos de importancia estratégica entre los que se pueden mencionar una serie de cambios en la gestión de la Autoridad Aeronáutica (DGAC, 2004) así como, en la mentalidad del mismo controlador.

La función de un controlador de tránsito aéreo está directamente relacionada con el resguardo de la vida de todas las personas que viajan vía aérea, de ahí la importancia de su labor y la urgencia de contar con una formación completa para la optimización de su desempeño. En la presente investigación se ha tomado como referentes principales el perfil y los ambientes de trabajo y de formación universitario que se aplican para el control de tránsito aéreo en un grupo representativo de centros de formación.

Esperamos que este trabajo de investigación sea retomado por la UNAH y la Agencia Hondureña de Aeronáutica Civil (AHAC) para asegurar a los controladores de tránsito aéreo una formación completa y constantemente actualizada para el desempeño de sus delicadas funciones.

\section{Método}

La consulta para la realización de esta investigación se hizo en los siguientes momentos: primero se hizo una revisión bibliográfica amplia de literatura sobre ATC, de la bibliografía consultada se priorizó aquella que se enfocaba directamente en temas de formación o bien que describía el ambiente de trabajo del ATC; seguidamente el trabajo se concentró en analizar aquellas fuentes que describían los perfiles de trabajo del Departamento de Navegación Aérea de la Agencia Hondureña de Aviación Civil, luego se hizo un repaso por un grupo de universidades y centros de formación que ofrecen la Carrera de ATC, haciendo énfasis en las que se localizan en los Estados Unidos de Norteamérica. Por último se aplicó una entrevista abierta a profesionales ATC o bien relacionados directamente con ATC en Honduras. De cada una de las fuentes mencionadas y del instrumento aplicado se sintetizan en el Tercer Capítulo una serie de hallazgos de los cuales se deducen cada una de las conclusiones propuestas. Además, en base a las orientaciones de los centros de formación estudiados, se hace una propuesta formativa que pueda 
incluirse dentro de una Licenciatura en Ciencias Aeronáuticas a impartirse dentro del Departamento de Ciencias Aeronáuticas de la FACES/UNAH.

\section{Resultados}

Esta parte de la investigación da cuenta de los principales aportes provenientes de los instrumentos aplicados y de las principales fuentes consultadas. En primer lugar se presentan los perfiles de puestos del Departamento de Navegación Aérea desde la Autoridad Aeronáutica, instancia desde la cual ésta cumple sus funciones de control de tránsito aéreo; luego se propondrán los datos extraídos de distintas fuentes que detallan los contenidos que se utilizan en la formación de controladores a un nivel secundario o universitario; por último se exponen las opiniones de profesionales encargados de dirigir el tránsito aéreo en Honduras y Centro América.

- Perfiles de puesto del Departamento de Navegación Aérea de la AHAC

El controlador aéreo concentra sobre sí una gran responsabilidad (Ontiveros, 2006), y lo hace en representación de la Autoridad Aeronáutica, la cual para asumir su responsabilidad distribuye las funciones de control de tránsito en 25 perfiles de puesto (DGAC, 2012), los cuáles marcan el ámbito en el cual podría ser empleado un egresado de un Técnico ATC de la UNAH. Los perfiles de puesto del Departamento de Navegación son los siguientes puestos de trabajo: 1. Jefe de Navegación Aérea, 2. Asistente de Navegación Aérea y Coordinador de Competencia Lingüística, 3. Coordinador de Seguridad Operacional ANS, 4. Inspector de los Servicios de Navegación Aérea, 5. Jefe Nacional de Tránsito Aéreo, 6. Sub Jefe Nacional de Tránsito Aéreo, 7. Coordinador Nacional de Búsqueda y Salvamento (SAR), 8. Jefe Regional de Tránsito Aéreo, 9. Supervisor Operativo Radar, 10. Supervisor Operativo Convencional, 11. Controlador Ejecutivo de Aproximación Radar, 12. Controlador Planificador de Aproximación Radar, 13. Controlador Ejecutivo de Aproximación Convencional, 14. Controlador Planificador de Aproximación, 15. Controlador Ejecutivo de Aeródromo, 16. Controlador Planificador de Control Tierra, 17. Jefe Nacional del Servicio de Información Aeronáutica, 18. Especialista MAP / PANS-OPS, 19. Especialista GIS, 20. Supervisor Regional del Servicio de Información Aeronáutica AIS/NOF, 21. Oficial de Información Aeronáutica (AIS/ARO/ NOF), 22. Oficial de Publicaciones de Información, 23. Jefe de Comunicaciones, Navegación y Vigilancia (CNS), 24. Técnico Electricista, Aires acondicionados y Grupos electrógenos, 25. Técnico Equipo Meteorológico Aeronáutico. 


\section{- Planes de Estudio para Controlador de Tránsito Aéreo}

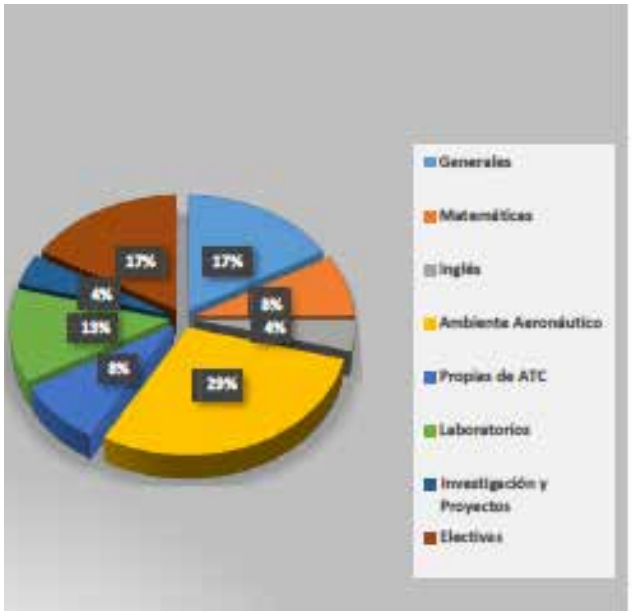

Gráfico 1: Community College del Condado de Baltimore



Gráfico 2: Institución de Aviación Broward College

Para efectos de comparación entre las distintas propuestas formativas de las Universidades e Institutos citados en este acápite, distribuimos las asignaturas de la siguiente manera: 1 . Generales, son las que ofrecen al estudiante conocimientos de formación general que le permitirán comprender mejor el resto de asignaturas que componen la carrera sea esta de grado universitario o del equivalente de educación secundaria, 2. Matemáticas, son las que facilitan al estudiante el conocimiento o la aplicación de conceptos abstractos matemáticos al ambiente

\footnotetext{
${ }^{1}$ Los datos para la elaboración de los gráficos presentados fueron extraídos de: College, B. (26 de octubre de 2014). Broward College. Obtenido de www- broward.edu/academics/programs/pages.

College, B. (26 de Octubre de 2014). Broward College. Obtenido de http://www.broward.edu/academics/programs/Program\%20Sheet\%20Library/A039.pdf) College, C. (20 de octubre de 2014). Comunity College. Obtenido de http://www.ccbcmd.edu/media/aviation/cti_handbook.pdf. College, H. (19 de Octubre de 2014). Hesston College. Obtenido de Hesston College:

http://www.hesston.edu/academics/departments/aviation/.

College, M. C. (16 de Octubre de 2014). Minneapolis Comunity and Tecnicals College. Obtenido de https:// mctc.decisionacademic.com/Catalog/ViewCatalog. aspx?pageid=viewcatalog\&topicgroupid=174\&topicid=8 3\&loaduseredits=False\&_ga=1.259511114.1390247888.1415199926.

College, M. S. (21 de Octubre de 2014). Mt. San Antonio College. Obtenido de http://www.mtsac.edu/presidentsboardreport/Air\%20Trafic\%20Articles\%20in\%20CC\%20Journal.pdf.
} 
aeronáutico y por ende al aprendizaje del control de tránsito aéreo, 3. Inglés, son asignaturas que ofrecen un conocimiento suficiente del Inglés general o bien la profundización del Inglés técnico aplicado a la aeronáutica, 4. Ambiente Aeronáutico, son las asignaturas que perteneciendo por ejemplo al ámbito propio de la aviación, complementan el desempeño en el campo de la aeronáutica, 5. Propias del ATC, son las asignaturas que tratan los temas específicos del control de tránsito aéreo como ser terminología propia, radares, comunicación, trabajo dentro de una torre de control, entre otros, 6 . Laboratorios, incluye los laboratorios propios de las asignaturas específicas de Control de Tránsito Aéreo, pero también todas aquellas que complementan algunas asignaturas teóricas, 7 . Investigación y proyectos, se trata de las asignaturas que preparan al estudiante para hacer investigación o para preparar proyectos de intervención, 8. Electivas, son todas aquellas que las universidades e institutos citados en esta investigación consideran como tales. Según la clasificación propuesta resultaron las siguientes combinaciones ${ }^{1}$ :

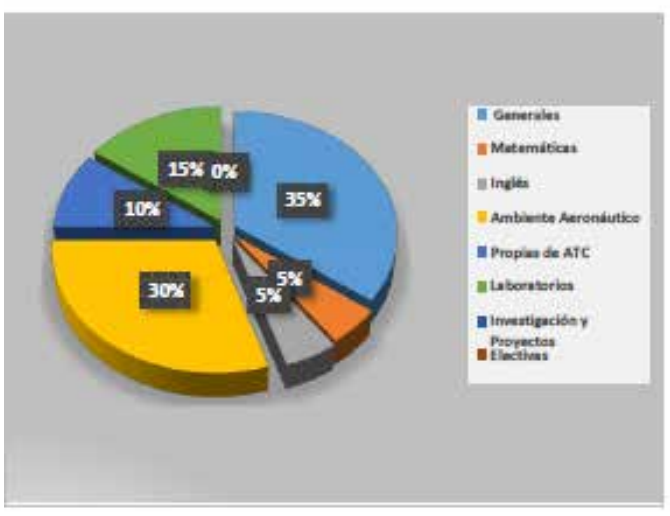

Gráfico 3: Hesston College

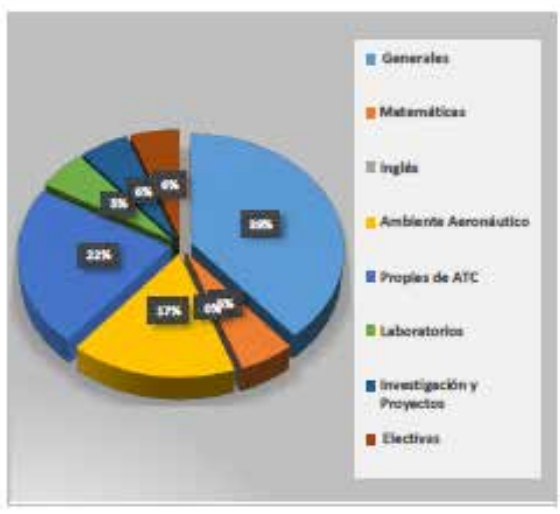

Gráfico 4: Hampton University 




Gráfico 5: Le Tourneau University

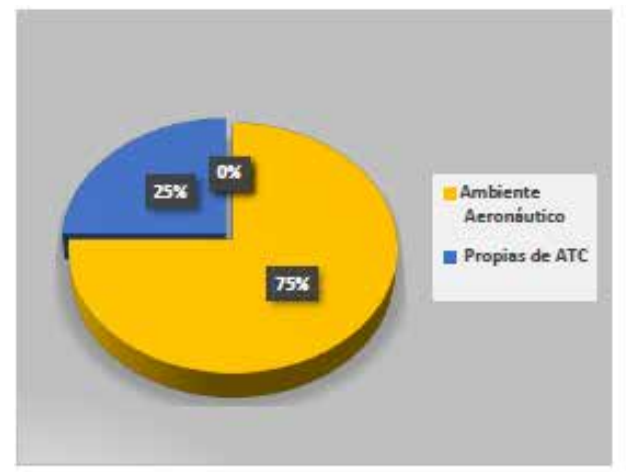

Gráfico 7: St. Cloud University State

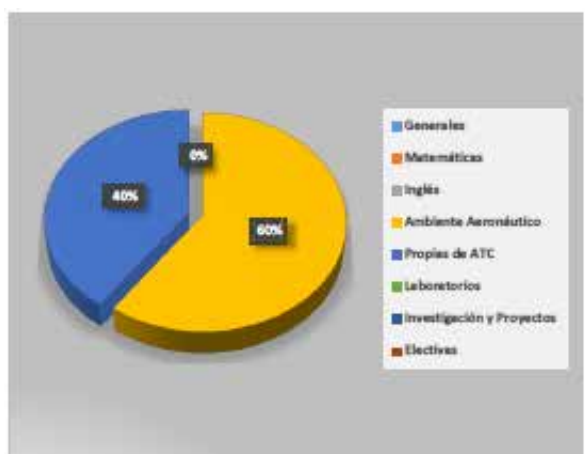

Gráfico 9: Academia Superior Aeronática

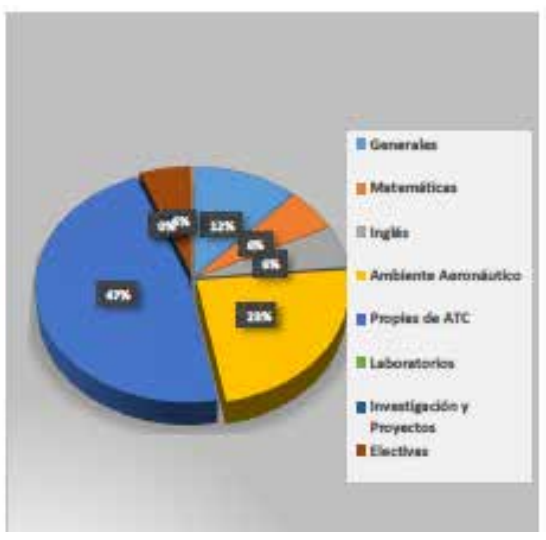

Gráfico 6: Minneapolis

Community and Tecnicals College

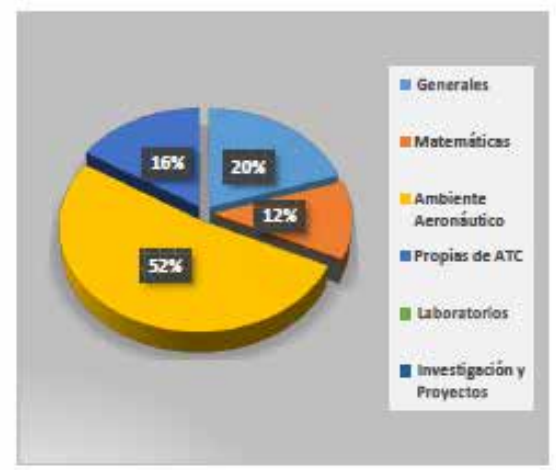

Gráfico 8: Mt. San Antonio College

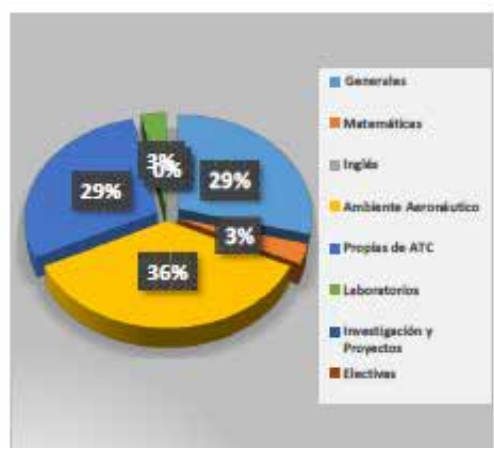

Gráfico 10: Universidad de Alaska, Anchorage 


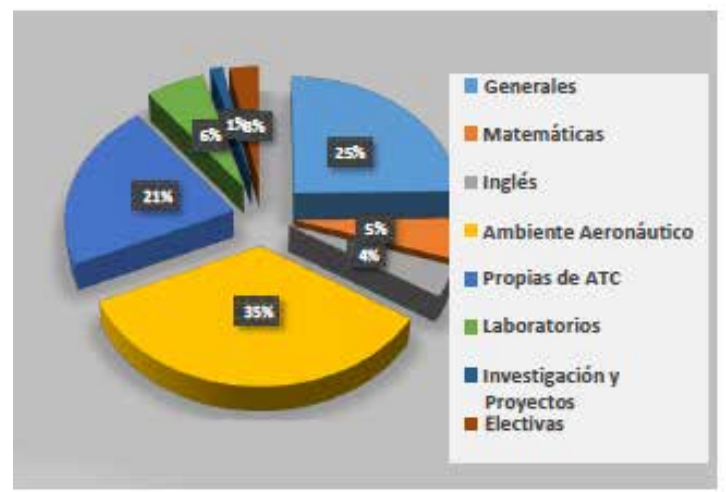

Gráfico 11: Resumen de todos los centros de formación.

- Consultas con los especialistas del campo aeronáutico

Esta parte se completó con los resultados de la consulta hecha a seis profesionales del área aeronáutica quienes juegan roles de facilitadores de servicios de controladores de tránsito aéreo o bien de usuarios del mismo. Se presenta un resumen de las conclusiones de las consultas hechas procurando extraer de las entrevistas la información relevante para orientar una propuesta formativa para ATC que dé cuenta de lo que está viviendo el controlador en el día a día. Las respuestas recolectadas se resumen en seis ítems que resumen un total de entre $12 \mathrm{y}$ 15 preguntas hechas a cada entrevistado:

\section{Cumplimiento de la regulación de la capacitación por parte de la Autoridad Aero- náutica}

- El cumplimiento del rol de la DGAC tiene una alta calificación y se entiende que no es un ente capacitador sino regulador del campo aeronáutico.

- Normalmente las Escuelas de Aviación conocen bien la reglamentación a ellas aplicable, esto debería facilitar la aplicación de la reglamentación por parte de la DGAC.

- Afecta negativamente el cumplimiento de la ley la inestabilidad laboral de los técnicos encargados.

- El tipo de examen hecho por la DGAC debería ir más allá de la memorización y debería adaptar mejor los exámenes que se toman del ambiente norteamericano. 
- Parte de la labor de la DGAC debe ser el de incentivar el campo aeronáutico, lo puede hacer por medio de una Ley de Incentivos.

- La contratación por padrinazgo político afecta negativamente el cumplimiento de las funciones de la DGAC.

- Parte de los examinadores de la DGAC son Ad-Honorem y no tienen una formación específica y exhaustiva para este campo.

- Los cobros de la DGAC se perciben como arbitrarios.

- Las capacidades técnicas de la DGAC se deben complementar con las capacidades académico-pedagógicas de la UNAH.

- La DGAC no continuó un proceso de capacitación de su personal que duró hasta 2005.

\section{En cuanto a actores y políticas de formación en materia aeronáutica}

- Una política de formación debe incluir temas como los aspectos a evaluar de los profesionales del campo y debe estar acompañada de una política de salarios; puede incluir una propuesta de Bachillerato Universitario.

- Se debería tomar en cuenta la tendencia mundial hacia la profesionalización de nivel universitario de los técnicos de aeronáuticos.

- El proceso de cambio en el campo aeronáutico debe venir de la DGAC y debe incluir a la UNAH y a otros actores del campo aeronáutico.

- Uno de los incentivos para las aerolíneas debería ser presentar a estas empresas los ahorros que puede representar para ellas los profesionales bien formados.

- La formación de técnicos aeronáuticos debe tomar en cuenta que estos no se pueden ausentar durante mucho tiempo de sus puestos de trabajo.

- Para una escuela de vuelo no influye mucho que en Honduras haya muchos aeródromos.

- Otra clave estratégica importante para la aeronáutica es despertar en la población el interés por este campo. 
- Los planes de capacitación de la DGAC deben establecerse de acuerdo a los servicios que se proponen prestar.

- Es crucial que la DGAC cuente con una planificación estratégica definida y estabilidad de sus empleados.

- El Sector aeronáutico está siendo atendido por profesionales que no fueron formados para este campo.

- Honduras puede ser un centro de despacho para la región centroamericana.

- La UNAH puede alianzas con países (EE UU, Israel, los demás de CA) e instituciones (FAA) para atender el campo aeronáutico.

- La UNAH puede aglutinar los actores de una política aeronáutica, sobre todo aquellos que pertenecen al Estado.

- El Norte de la formación debe ser la Categorización del País.

\section{Características de los procesos de formación}

- Es imprescindible en el proceso de formación la capacitación en el puesto de trabajo, dicha dinámica en el caso de la DGAC es la principal forma de capacitación.

- Prevalece una mayor importancia para la formación técnica básica de nivel secundario.

- Se puede formar Técnicos Universitarios.

- La dispersión en la que se da la formación hace que no se vea clara la relación entre las distintas partes que conforman los contenidos.

- Se tiene la impresión de que los planes de formación no están conectados con las necesidades reales del campo.

- Lo normal es que el personal aeronáutico tenga sólo grado de técnico aun en el caso de los pilotos.

- La Formación es en Cursos, sin un diseño, está supeditada a lo que se va a estudiar (normalmente al equipo). 
- Se ha aprovechado la formación recibida pero de forma aislada: pilotos y técnicos.

- Cada quien es capacitado para la situación específica en la que va a trabajar o para el equipo que va a operar.

\section{Criterios a respetar en la selección de contenidos}

- Prevalece la dispersión en la capacitación que se recibe y un síntoma de ello la brevedad de cada uno de los talleres ofrecidos.

- Una formación común para técnicos y administrativos debe ser el lenguaje técnico propio del campo.

- Hasta ahora los contenidos de capacitación los escoge ICCAE con poca o ninguna participación de la Autoridad Aeronáutica.

- Entre las materias prioritarias a enseñar están: Matemáticas, Física, Geometría, Cultura General, Generalidades de la Aviación.

- La definición de contenidos debe respetar el equipo del que se dispone y los objetivos que se buscan al formar al personal.

- Parte de los contenidos de formación deberían ser enseñar las tecnologías de punta aplicadas a la aeronáutica.

- Es necesario formarse en Inglés Aeronáutico, físico-matemática, computación y manejo de páginas web sobre temas aeronáuticos.

- Son prioridades de contenidos la Ley de Aeronáutica Civil, RACs, conocimiento de los aviones que aterricen en Honduras.

- Otros temas importantes: Factores Humanos, físico-matemáticas, estructuras de aeronaves.

\section{Características de los capacitadores}

- Hay coincidencia en pedir que los capacitadores sean expertos en su campo.

- La formación hasta ahora la ha venido ofreciendo personal con nivel técnico y se cuenta personas para el rubro formativo, de nivel internacional. 
- La UNAH requiere de una certificación como formador en el campo aeronáutico.

\section{Comentarios sobre la labor de los Controladores de Tránsito directamente}

- Algunas carencias de los ATC son: Fechas no definidas de formación/ausencia de recurrentes/falta de formación complementaria del campo de ATC/la formación es casi exclusiva en el lugar de trabajo.

- Casi todo el personal de Control de Tránsito Aéreo tiene alguna formación universitaria y algunos de ellos el grado universitario.

- Para seleccionar contenidos se debería hacer evaluaciones en base a los perfiles y atribuciones existentes.

- Dentro de la formación requerida se debe incluir la formación sicológica para los Jefes.

- El perfil de controlador debería incluir: estabilidad sicológica, capacidad para atender tareas simultáneas, madurez en la toma de decisiones, edad para iniciar el trabajo entre 23 y 28 años, edad para finalizar 48 años, no padecer de hipertensión, disponibilidad para hacer revisiones periódicas de la condición sicológica.

- En TNT, no se percibe un tráfico aéreo demasiado estresante.

- Parte importante del trabajo de un ATC es la coordinación con otros ATC.

- Los horarios de mayor tráfico en TNT son de 11:00AM a la 1:00 PM.

- Propuesta formativa

En todas la fases de esta investigación, se han tomado en consideración y se han conjugado de manera directa o indirecta tres elementos, la necesidad de contar en Honduras con un grupo de controladores que cuenten con una formación completa y actualizada para el desarrollo de sus funciones, la participación de la UNAH en la selección y composición de los contenidos más acordes para conformar una propuesta formativa en el más alto nivel educativo y, el examen de varias currículas formativas que ya están aplicándose. Partiendo de los puntos mencionados esta investigación propone las siguientes asignaturas para la formación de una Controlador de Tránsito Aéreo dentro de una Licenciatura en Ciencias Aeronáuticas: 


\begin{tabular}{|c|c|c|c|}
\hline \multicolumn{4}{|c|}{ Técnico Controlador de Tránsito Aéreo } \\
\hline Tipo de asignatura & Código & Asignatura & UV \\
\hline \multirow[t]{5}{*}{ Generales } & EG-011 & Español General & 4 \\
\hline & DAE-205 & Computación I & 3 \\
\hline & $\mathrm{HH}-101$ & Historia de Honduras & 4 \\
\hline & $S C-101$ & Sociología & 4 \\
\hline & No definido & Electiva de Cultura Física y Deportes o Arte & 3 \\
\hline \multirow[t]{5}{*}{ Físico-Matemáticas } & MM-110 & Matemáticas I & 5 \\
\hline & $\mathrm{MM}-111$ & Geometría y Trigonometría & 5 \\
\hline & $\mathrm{MM}-201$ & Cálculo I & 5 \\
\hline & $\mathrm{MM}-211$ & Vectores y Matrices & 3 \\
\hline & $\mathrm{FS}-100$ & Física General I & 5 \\
\hline \multirow[t]{5}{*}{ Inglés } & IN-101 & Ingles I & 4 \\
\hline & IN-102 & Ingles II & 4 \\
\hline & $\mathrm{IN}-103$ & Ingles III & 4 \\
\hline & IN-104 & Ingles IV & 4 \\
\hline & CAG-055 & Ingles Aeronáutico & 4 \\
\hline \multirow[t]{4}{*}{ Ambiente Aeronáutico } & CAG-011 & Introducción a la Aviación & 2 \\
\hline & CAG - 022 & Meteorología & 4 \\
\hline & CAG - 033 & Factores Humanos I & 3 \\
\hline & CAG - 044 & Factores Humanos II & 3 \\
\hline \multirow[t]{4}{*}{ Propias CTA } & No definido & Controlador de Tránsito Aéreo I & 3 \\
\hline & No definido & Controlador de Tránsito Aéreo II & 3 \\
\hline & No definido & Controlador de Tránsito Aéreo III & 4 \\
\hline & No definido & Controlador de Tránsito Aéreo IV & 4 \\
\hline \multirow[t]{5}{*}{ Laboratorios CTA } & No definido & Laboratorio de CTA I & 2 \\
\hline & No definido & Laboratorio de CTA II & 2 \\
\hline & No definido & Laboratorio CTA III & 2 \\
\hline & No definido & Laboratorio CTA IV & 2 \\
\hline & \multicolumn{2}{|c|}{ TOTAL UNIDADES VALORATIVAS } & 95 \\
\hline
\end{tabular}

Tabla 1: Asignaturas para la formación de un Controlador de Tránsito Aéreo 




Gráfico 12: Técnico CTA / Propuesta DCA-FACES-UNAH

\section{Conclusiones}

La formación de los ATC hondureños según una perspectiva de País corresponde a la Autoridad Aeronáutica, la cual hasta ahora ha sostenido una relación más bien esporádica con la UNAH. Las conclusiones que se ofrecen a continuación han sido pensadas para fortalecer esta relación de manera que se enfatice la condición de Autoridad en materia de formación para controladores de tránsito aéreo de la Agencia Hondureña de Aeronáutica Civil. Luego de examinar los perfiles de puesto en materia de control de tránsito aéreo, hacer un recorrido por la oferta académica de diez centros de formación y de consultar a un grupo de profesionales comprometidos con la aeronáutica en Honduras se puede concluir en lo siguiente:

La tradición del control de tránsito aéreo tiene cerca de un siglo y en su proceso ha evolucionado de manera significativa, estos avances no han llegado con la fuerza y relevancia que se esperaría a nuestro País, en parte por la falta de formación de los ATC de la AHAC que han venido siendo formados sin referentes a los progresos tecnológicos logrados por el control de tránsito a nivel mundial.

La Ley que dio origen a la Dirección General de Aeronáutica Civil es suficiente en las atribuciones que le asigna a esta dependencia con respecto al control del tránsito aéreo; es de esperarse que la coyuntura presente en la que esta Dirección se está convirtiendo en la Agencia Hondureña de Aeronáutica Civil, no provoque la pérdida de estas prerrogativas que por derecho le pertenecen a la Autoridad Aeronáutica. 
Honduras no tiene un tráfico aéreo que pueda provocar niveles de stress extremos, sin embargo, las condiciones en las que se ejerce esta labor están vinculadas directamente con el resguardo de la vida física de las personas y su atención es un ejercicio cotidiano de soberanía y de rescate la importancia del ejercicio de la autoridad en el campo aeronáutico, de ahí que sea necesario prestar una mejor atención a los controladores en cuanto a su formación inicial y actualización de conocimientos y destrezas.

El desarrollo de los perfiles del personal del Departamento de Navegación Aérea está bastante diversificado y ofrece una base suficiente para la generar una política de formación de acuerdo a las funciones de cada empleado. Entre las prioridades de esta política debería estar una revisión de los perfiles mencionados que dé cuente de la labor desempeñada por cada uno de los cargos existentes para distinguir entre éstos cuáles son más necesarios, cuáles se pueden fusionar, cuáles tienen cargas mayores a las que puedan atender, entre otros aspectos.

Considerando la importancia estratégica de los servicios de tránsito aéreo, el Estado debe examinar estas funciones en términos de costo beneficio, en base a lo cual puede resultar necesario formar al personal para el desarrollo de servicios con criterios de calidad; como producto de una mejoría de los servicios debería aplicarse de manera ordenada y justa, los cobros correspondientes por la misma AHAC y destinarlos a su sostenibilidad; contando con los fondos recaudados por servicios se puede elaborar una política salarial que pueda ir aparejada a los niveles de formación de los empleados dentro de la institución.

Al momento de explicar su trabajo y la problemática cotidiana, los controladores reflejan un conocimiento suficiente de su labor técnica, no así de los fundamentos estratégicos y de razón de ser de su labor; esta situación podría estarse dando por la falta de una formación que incentive la investigación y el espíritu crítico, valores intelectuales que pueden ser aportados por la UNAH dentro de un proceso formativo inspirado en el modelo educativo de esta casa de estudio.

La composición de asignaturas de las carreras examinadas coinciden con el interés mostrado por los entrevistados por una formación que incluya una base amplia de formación general, esta formación debería responder, como efectivamente responde a dos aspectos, el de ofrecer una cultura general (dada por las asignaturas generales) y un conocimiento suficiente del ambiente aeronáutico (dado por las asignaturas propias del ambiente aeronáutico). 
Como se muestra con las instituciones reseñadas, en la mayoría de casos la Carrera de Controlador de Tránsito Aéreo se ofrece como un Técnico Universitario; con esto los hallazgos coinciden con dos elementos resaltados en la investigación: la consideración de los entrevistados de que la demanda de formación se puede atender desde un Técnico Universitario y la apertura del Modelo Educativo de la UNAH que ofrece la oportunidad de contar en las Licenciaturas con salidas intermedias de Técnicos Superiores.

Una Carrera de Técnico en Control de Tránsito Aéreo ofrecida desde la UNAH fortalecería ostensiblemente, muchas de las funciones desempeñadas por los controladores en sus labores cotidianas. Pero además, les permitiría conocer con mayor profundidad el propio campo y proponerse temas de investigación para la mejora del propio desempeño y el mayor empoderamiento de la función reguladora del Estado.

La carencia de formación universitaria entre los ATC tiene como una de sus consecuencias una representación con limitaciones del País en los espacios de análisis y formación para ATC que hasta ahora son todos o la mayoría de ellos de índole regional o internacional; de manera que una formación con grado universitario en el mediano plazo puede mejorar el estatus y los aportes de Honduras al mundo del control de tránsito.

La LCA es una oportunidad única para proponer un Técnico en Control de Tránsito Aéreo puesto que permitiría a quiénes ya están trabajando como ATC obtener su titulación de técnico universitario y les pondría a la mitad de la Licenciatura, abriéndoles con esto nuevas posibilidades de empleo y dándoles la oportunidad de hacer carrera administrativa dentro de la AHAC o en alguna otra institución del estado relacionada con el rubro aeronáutico.

Un tema práctico que queda pendiente de profundizar es el de investigar cuáles de las universidades y centros de formación examinados tienen vínculos fuertes con el Gobierno de los Estados Unidos, de manera que éste les pueda solicitar colaboración con el Estado de Honduras para dar asesoría y formación a nuestros controladores en base a los currículos desarrollados por estas instituciones.

Es un lugar común entre los entrevistados que persiste la fragmentación en los procesos de capacitación con los que se atiende la formación de los Controladores de Tránsito en Honduras, de hecho no se recogen evidencias que delimiten 
los contenidos teóricos de los contenidos prácticos y no se cuenta con un cuerpo de contenidos, metodologías y perfiles de capacitadores en poder la AHAC.

Dado que no existe una política de formación definida por parte de la Autoridad Aeronáutica, los contenidos de formación, los tiempos en que ésta debe desarrollarse, los espacios físicos para el aprendizaje y la filosofía pedagógica, siguen siendo determinados por el Instituto Centroamericano de Capacitación Aeronáutica (ICCAE), dependencia de COCESNA.

La formación básica para el desarrollo de las funciones de control de tránsito es recibida predominantemente en el lugar de trabajo o bien mediante cursos que oscilan entre una y ocho semanas; considerando que estos procesos formativos son asumidos con sentido de responsabilidad por el personal capacitado, se puede decir que ofrecen una buena base para el desempeño de las funciones de control. Sin embargo, continúa siendo necesario y en algunos casos urgente el que los controladores reciban los cursos de formación recurrente que les permitan actualizar sus conocimientos; esta renovación constante es un imperativo en el mundo de la aeronáutica que cambia constantemente.

Se considera que la Autoridad Aeronáutica cumple con sus funciones en lo que respecta a la supervisión de los procesos de formación y en cuanto a la certificación de los mismos; sin embargo persisten dificultades para asumir de una mejor manera esta labor, entre ellas se mencionan la falta de una planificación estratégica de la formación, la inestabilidad laboral de los empleados, el sobredimensionamiento del personal administrativo, la prevalencia del clientelismo político al momento de hacer los nombramientos, entre otros.

Como mencionó una de las personas entrevistadas, no basta con formar al Controlador en las materias que pertenecen estrictamente al Control de Tránsito Aéreo, es necesario ofrecerle formación del campo sicológico que le permita hacer su trabajo evitando el estrés excesivo y conservando la salud mental. Una forma de complementar esta formación deberían ser las evaluaciones sicológicas periódicas que den cuenta de la idoneidad del empleado para continuar ejerciendo sus labores cotidianas.

Corresponde a la institucionalidad del Estado, en este caso a la AHAC y a la UNAH, liderar una política formativa en materia aeronáutica particularmente en lo relacionado con el Control de Tránsito Aéreo; la AHAC debe aportar todo la experiencia técnica acumulada durante más de medio Siglo de regulación de la Aeronáutica en Honduras, por su parte la UNAH debe incorporar los componentes 
académico-pedagógicos que requiere esta misma política para formar controladores que trasciendan el quehacer técnico generando capacidades para la crítica, la autocrítica y la investigación científica.

- En un espectro amplio una política de formación para los ATC hondureños, puede estar compuesta de los siguientes elementos:

- Contenidos, los cuales deben ser definidos por la Autoridad Aeronáutica en colaboración con la UNAH.

- Sujetos a capacitar; en este caso la población principal y casi única sería el personal de la AHAC, especialmente los empleados del Departamento de Navegación Aérea.

- capacitadores, para los cuales se debe diseñar un perfil que combine la formación académica adecuada con la experiencia del campo del control de tránsito.

- Procesos de formación, que deberán ser diseñados y planificados estratégicamente tomando en cuenta la condición específica de los ATC (disposición de tiempo, condiciones de trabajo, temas prioritarios de formación, espacios de aprendizaje propios, entre otros).

- Recursos, incluyendo en primer lugar los que pueden ser generados por la venta de los servicios prestados por la AHAC, pero sin excluir los recursos técnicos y financieros que se puedan obtener por medio de la cooperación internacional y regional.

- La inclusión de una Técnico en Control de Tránsito Aéreo en oferta académica del Departamento de Ciencias Aeronáuticas, requiere de la sanción de un convenio específico entre la AHAC y al UNAH, dicho convenio debería cubrir aspectos como la certificación de los egresados como ATC, la utilización de las capacidades instaladas de la AHAC para el entrenamiento de los estudiantes incluyendo torre de control y control terrestre, uso del equipo de aproximación o equipo de simulación de la aproximación, entre otros. 


\section{Bibliografía}

- DGAC. (2004). Ley de Aeronáutica Civil. Tegucigalpa: DGAC.

- DGAC. (2012). Manual de Funciones y Atribuciones del Personal. Tegucigalpa: DGAC.

- College, B. (26 de octubre de 2014). Broward College. Obtenido de wwwbroward.edu/academics/programs/pages.

- College, B. (26 de Octubre de 2014). Broward College. Obtenido de http://www. broward.edu/academics/programs/Program\%20Sheet\%20Library/A039.pdf)

- College, C. (20 de octubre de 2014). Comunity College. Obtenido de http:/l www.ccbcmd.edu/media/aviation/cti_handbook.pdf.

- College, H. (19 de Octubre de 2014). Hesston College. Obtenido de Hesston College: http://www.hesston.edu/academics/departments/aviation/.

- College, M. C. (16 de Octubre de 2014). Minneapolis Comunity and Tecnicals College. Obtenido de https://mctc.decisionacademic.com/Catalog/ViewCatalog.aspx? pageid=viewcatalog\&topicgroupid=174\&topicid=83\&loaduseredits=False\&_ga=1.259511114.1390247888.1415199926.

- College, M. S. (21 de Octubre de 2014). Mt. San Antonio College. Obtenido de http://www.mtsac.edu/presidentsboardreport/Air\%20Trafic\%20Articles\%20 in\%20CC\%20Journal.pdf.

- Ontiveros, J. (2006). Descubrir el Control Aéreo. Madrid: Aeropuertos Españoles y Navegación Aerea. AENA. 